\title{
EMERGING PSYCHEDELIC-ASSISTED THERAPIES: IMPLICATIONS FOR NURSING PRACTICE
}

Dominique Denis-Lalonde BN RN, MN Candidate and Andrew Estefan, PhD

Faculty of Nursing, University of Calgary

Corresponding author: ddenislalonde@ucalgary.ca

\begin{abstract}
Background

Psychedelic-assisted therapy research is demonstrating unprecedented rates of success in treating mental illness, addictions, and end-of-life distress. This psychedelic renaissance is a turning point in how complex human conditions can be treated and has implications for nursing knowledge, advocacy, and practice internationally.
\end{abstract}

\section{Objective}

This article aims to explore the current state of knowledge in the field of psychedelic-assisted therapy and the practice implications for nurses.

\section{Methods}

A scoping review of the literature was undertaken with a focus on mental health, addictions, and palliative care indications. Commentaries, syntheses, and reviews from the last 20 years were included, as well as all relevant primary study results. We then explored what is known about the nurse's past and present role in this field.

\section{Results}

The nurse's role in psychedelic-assisted therapy and research has been hitherto mostly invisible and thus remains under-explored and undefined. The profession is ideally positioned, however, to contribute to the future of this promising field.

\section{Conclusion}

As advocates for safe, ethical, and interdisciplinary practice, nurses can lead the development of psychedelicassisted therapy practice, ethics, research, advocacy, policy, and education. This article provides guidance and support for prescient nursing leadership in these areas.

Keywords: addiction, drug therapies, mental health promotion, palliative care, psychotherapy 
For thousands of years, psychoactive drugs have been used throughout the world for the promotion of human health and wellbeing. Their history is long and tumultuous, and from the 1950s to the early 1970s, psychedelics were being researched as possible treatments for a wide variety of indications. ${ }^{1}$ The uptake of psychedelic substances within controversial countercultures of the late 1960 s contributed to a politicallycharged, media-induced "moral panic." Psychedelic drugs were subsequently made illegal in the U.S., Canada, and all over the world. A short time later, in the 1970s, the war on drugs began. Social pressures and legal prohibition contributed to the cessation of human psychedelic research. An ongoing inquiry into the development of psychedelic-assisted therapies was no longer viable. For decades, research into psychedelic drugs as treatment has laid mostly dormant.

Recently, interest has re-emerged in the contribution that psychedelic drugs can make to alleviating the suffering associated with various mental health problems. In the late 1970s through to the turn of the century, the idea that psychotherapy accompanied by a single dose of psilocybin (found in "magic mushrooms") could provide substantial relief for treatment-resistant depression would have been far-fetched. Today, this idea is gaining traction and there is more psychedelic research happening than ever before. ${ }^{2}$ Research into psychedelic drug use in treatment has offered significant insights into brain function and the human mind, ${ }^{3}$ and is demonstrating unprecedented rates of success in alleviating mental health problems, end-of-life distress, and substance use disorders. Although some of the research findings are still preliminary, evidence for the effectiveness of psychedelic drugs is mounting. As a result, several therapies have been granted 'breakthrough' status for hard-to-treat conditions.

In the coming decade, nurses and other care providers will be expected to be knowledgeable about emerging psychedelic therapies. In particular, nurses will be positioned to play a critical role in research, treatment development, and service provision of psychedelic-assisted therapies. In this article, we summarize the literature and discuss nurses' hitherto invisible but crucial role in this field. Having explored the history of psychedelic therapies and nurses' roles in the provision of these therapies, we argue how nurses are suited to lead, advocate, research, innovate, and practice in what has been termed the psychedelic renaissance.

\section{METHOD}

In this discursive scoping review, we argue that treatment options for complex human conditions are at a turning point as a result of ongoing developments in psychedelic drug research. A literature review was undertaken with a focus on mental health, addictions, and palliative care indications. Commentaries, syntheses, and reviews from the last 20 years were included, as well as all relevant primary study results. By checking reference lists and having informal conversations with experts in the field, we also explored what is known about the nurse's past and present role in this field.

\section{PSYCHEDELIC-ASSISTED THERAPY AS AN EMERGING PRACTICE}

The term 'psychedelic' (meaning 'mind manifesting' or 'mind revealing') was coined in Weyburn, Saskatchewan by English psychiatrist and researcher Humphry Osmond in $1957 .{ }^{4}$ Osmond used lysergic acid diethylamine, also known as LSD or 'acid,' in experimental research that attempted to understand the experience of schizophrenia. In the course of his research and that of his colleagues internationally, many important contributions were made to modern psychiatric theory, such as the discovery that neurotransmitter events in the brain may correspond to changes in behavior. ${ }^{5}$ Osmond also became interested in his research participants' reports that their psychedelic experiences were meaningful and had a lasting, positive impact on their mental health. He began to explore how LSD might be used as a therapeutic agent for people with alcoholism. Osmond received significant community and institutional support for this work which was claimed to have a $40-45 \%$ success rate one year after treatment. ${ }^{4,6,7}$ Meanwhile, similar scientific experimentation was occurring internationally, and psychedelic substances were being used to understand and ostensibly treat alcohol dependence and a variety of mood disorders. ${ }^{8}$ Some of these treatments showed considerable promise until support for psychedelic research was withdrawn and

J Mental Health Addic Nurs Vol 4(1):e1-e13; February 19, 2020.

This article is distributed under the terms of the Creative Commons Attribution-Non

Commercial 4.0 International License. (c) Denis-Lalonde and Estefan. 
the drugs were designated schedule I. Alongside the sociopolitical shifts of the time, psychedelic substances were believed to be dangerous, without therapeutic or medical value, and with a high potential for abuse.

At the turn of the 21st century, psychedelic researchers and therapists strongly emphasized rigorous scientific methodologies. The construction of the clinical 'set' and 'setting' was constructed to address stigmatizing sociopolitical discourse about illegal drugs and to mitigate risks to study participants. 'Set' refers to the individual's inner world and psychological goals going into the treatment, whereas 'setting' is their outer world which includes the cultural, social, and physical environments in which the experience(s) will take place. ${ }^{9}$ The construction of 'set and setting' begins with comprehensive screening, including a detailed history, physical and psychological assessments, with strict eligibility criteria. If the candidate is eligible for the study and the treatment is deemed appropriate, the participant (or client) will typically undergo several psychotherapy or talk therapy sessions with one or two trained practitioners before the drug-facilitated session(s), as well as a series of integration sessions following the psychedelic experience. Attention to the substance selected, the dose, the 'set and setting', and the development of a therapeutic alliance between the practitioner(s) and the participant is considered critical to achieving the best outcomes and reducing the risk of adverse events. ${ }^{10,11}$

During the psychedelic-assisted session, participants receive a dose of a drug, eyeshades, a comfortable room and place to lie down, and music to promote relaxation and introspection. They are encouraged to 'let go' and explore the thoughts and emotions that may arise during the experience. Participants are monitored and closely supported by their practitioner(s) for the duration of the session, which can last from a few hours to an overnight stay.

Participants frequently struggle to describe their experiences, though terms like mystical, spiritual, transcendental, interconnected, oneness, selfactualization, and existential awe have characterized their descriptions (e.g. ${ }^{12,13}$ ). Researchers have noted an association between these 'mystical-type' experiences and "abrupt, substantial, and sustained changes in behavior and perception." "14 Though psychedelics may have pharmacotherapeutic effects by themselves, the benefits seem to be maximized in an intentional, structured intervention delivered by trained practitioners. ${ }^{15}$ These practitioners have included psychiatrists, psychotherapists, psychologists, physicians, social workers, nurses, and other support staff. As such, the therapeutic process and its outcomes are augmented or "assisted" by psychedelics rather than being merely the result of the drugs' pharmacological actions alone.

In their guidelines for minimizing adverse reactions, Johnson, Richards, and Griffiths ${ }^{16}$ provided a detailed description of the risks associated with human psychedelic research. They concluded that these therapies are well-tolerated by most participants and unwanted side effects are most often short and resolved by the end of the session. Safe use of psychedelics in controlled settings is well-documented and demonstrates a strong benefit to risk ratio (e.g. ${ }^{12,17,18}$ ). Though serious adverse events are uncommon, they can potentially include physiological toxicity, abuse and dependence, acute psychological distress and dangerous behavior, prolonged psychosis, and lasting perceptual abnormalities. ${ }^{16}$ Johnson et al. ${ }^{16}$ provided detailed safety guidelines "intended to support the safe administration of high doses of [psychedelics] to human volunteers while minimizing potential adverse reactions." ${ }^{\text {"16 }}$ To conduct psychedelic research as safely as possible, they also recommended that particular attention be given to the selection of volunteers (physical and psychiatric screening), appropriate selection and training of study personnel, the physical environment of the session, the preparation of the volunteers (including education, consent, and development of trust), the supervision and psychological support available during the session, and post-session follow-up. Although most psychedelic-assisted therapies and research currently focus on strategies for individual healing and well-being, ceremonial use in group settings dates back centuries and also warrants investigation as a cost-effective and potentially efficacious treatment model. ${ }^{19}$

Psychotherapy and conventional pharmaceutical treatments, whether in isolation or combined, are often not sufficient to treat chronic and persistent mental illness. ${ }^{20}$ Psychedelic-assisted therapy combines some form of psychotherapy protocol with the administration of a potent psychoactive substance in a curated and

J Mental Health Addic Nurs Vol 4(1):e1-e13; February 19, 2020.

This article is distributed under the terms of the Creative Commons Attribution-Non Commercial 4.0 International License. (c) Denis-Lalonde and Estefan. 
supervised 'set and setting.' In this treatment context, results appear to be beneficial in a high proportion of cases and particularly for those who are treatmentresistant. $^{3,21}$ The entire therapeutic protocol, which may last weeks to months, is understandably resourceintensive. Unlike other mainstream psychiatric interventions and treatments, psychedelic-assisted therapy does not require daily use of a drug, nor is treatment expected to be ongoing over the lifetime. This marks a significant shift from conventional pharmacological treatments for mental illness.

\section{PSYCHEDELIC RESEARCH AND NURSING PRACTICE}

Although nurses have participated in research, development, and provision of psychedelic-assisted therapy, their role and contribution to the field remain largely invisible. In the acknowledgements section of his 345-page book describing his 1990s research with N,N-dimethyltryptamine (DMT), Strassman ${ }^{22}$ somewhat paternalistically stated that nurses "provided heroic, cheerful, and disciplined [...] support for all the studies." 22 One of the nurses in these studies had previously commented that "while clinical writing related to psychedelic nursing is relatively meager throughout the ' $60 \mathrm{~s}$, writing by nurses about their experiences during the thousands of hours of intensive psychedelic research is virtually non-existent. No nurses, to the best of my knowledge, served as coauthors on published research papers, and no narrative or journal notes by nurses working in psychedelic studies are available." 23

It seems little has changed in 25 years. Nurses Rosa, Hope, and Matzo ${ }^{24}$ noted that while other professions such as psychology, medicine, and pharmacology have disseminated the outcomes of their psychedelic research, nursing has not, and to the best of our knowledge, there are no nurse-led psychedelic studies to date, that is, where the primary investigator is a nurse.

A closer look at the literature reveals something of the role of the nurse in psychedelic research. In the first 3,4-methylenedioxymethamphetamine (MDMA)assisted trial for post-traumatic stress disorder (PTSD), the co-therapist team was comprised of a psychiatrist and a psychiatric nurse, and a nurse was required to be onsite for all experimental sessions as well as for overnight stays. ${ }^{25}$ Nurses were explicitly mentioned as therapists or in other supportive roles in several other studies, including research with psilocybin, ${ }^{24} \mathrm{LSD},{ }^{4,6}$ $\mathrm{DMT}_{,}{ }^{22}$ and ketamine. ${ }^{26}$ In all recent peer-reviewed studies and clinical trials, research participants are comprehensively assessed before treatment as well as monitored during and after treatments, which may include blood tests, vital signs, electrocardiograms, urine tests, breathalyzers, pregnancy tests, physical examination, MRI, and/or other assessments. It is reasonable to assume that where nurses were involved in studies, they were also part of the screening and data collection process.

In the re-emerging field of psychedelic-assisted therapy, there is evidence that nurses' roles can be expanded. In the first comprehensive guidelines and competencies for the training of psychedelic therapists, Phelps ${ }^{11}$ listed nurses as appropriate candidates for this work. Phelps ${ }^{11}$ also developed a certificate program in psychedelic-assisted therapies and research which has been available at the California Institute of Integral Studies since $2016 .{ }^{27}$ The program lists registered nurses and nurse practitioners as eligible applicants, and several cohorts to date have included nurses. Though there is recent evidence that a handful of nurses and nurse practitioners are becoming more visibly involved in psychedelic-assisted therapy, many more may be interested in research and practice in this field.

\section{INDICATIONS FOR PSYCHEDELIC- ASSISTED THERAPIES}

Currently, psychedelic-assisted therapies are primarily indicated for chronic, complex, and difficult-to-treat conditions that have not improved with conventional pharmacological and non-pharmacological approaches. To review the evidence without becoming entangled in the complex and at times inconclusive neuropharmacology, we discuss several psychoactive substances under the umbrella of psychedelics. Though they all elicit strong psychological and perceptual effects at high doses, only some of them are "classic" psychedelics which typically denote compounds with specific agonist activity at the serotonin $2 \mathrm{~A}$ receptor site. ${ }^{14}$ As a comprehensive review of the pharmacological literature is beyond the scope of this article, we offer a summary of common psychedelics and their therapeutic applications. 


\section{MDMA and PTSD}

Possibly the most publicized research and application of psychedelic-assisted therapy is the use of 3,4-methylenedioxymethamphetamine (MDMA, a synthesized compound commonly known as ecstasy or molly) for the treatment of post-traumatic stress disorder (PTSD). In the first randomized controlled pilot study of MDMA to treat PTSD, $83 \%(n=12)$ of participants in the active treatment group (MDMA-assisted psychotherapy) no longer met the DSM-IV diagnostic criteria for PTSD compared to $25 \%(n=8)$ in the psychotherapy-only group. ${ }^{25}$ In Ot'alora et al.'s ${ }^{17}$ phase 2 randomized controlled trial, participants who had received at least one active dose of MDMA ( $n=25)$ alongside psychotherapy reported a significant reduction from baseline in PTSD symptoms such as depression, poor sleep quality, dissociation, and suicidal ideation $(p<.001)$. Notably, $76 \%$ of participants in the intervention group no longer met the diagnostic criteria for PTSD 12-month after the treatment. No medical interventions were needed for the expected but temporary increases in heart rate and blood pressure and all adverse reactions reported during treatment were mild to moderate and short in duration, with the most common being anxiety $(60 \%)$ and jaw-clenching (64\%). The treatment protocol was well tolerated by participants and demonstrated a sustained positive effect of MDMA-assisted therapy for individuals with chronic PTSD. ${ }^{17}$ In addition to this evidence, several case studies have suggested MDMA-assisted therapy can have a significant impact on reducing troubling symptoms for people struggling with debilitating PTSD. ${ }^{28,29}$

As a result of these findings, the U.S. Food and Drug Administration (FDA) granted Breakthrough Therapy designation to MDMA-assisted therapy for PTSD in 2017 to expedite drug development. ${ }^{30} \mathrm{~A}$ randomized, double-blind, placebo-controlled, multi-site phase 3 clinical trial is currently recruiting participants with severe PTSD, including sites in Vancouver and Montreal, with an anticipated sample size of 200300 participants. ${ }^{30}$ If previous promising results are replicated in phase 3 trials, MDMA-assisted therapy will become an FDA-approved treatment option for people suffering from PTSD.

\section{Psilocybin and Depression}

Psilocybin is a psychoactive compound that, while found in over 100 species of mushrooms, can also be synthesized. ${ }^{21,31}$ Preliminary evidence suggests that psilocybin may be a better option to currently available therapies for depression and has accordingly been granted Breakthrough Therapy status by the FDA for treatment-resistant depression ${ }^{32}$ and major depressive disorder. ${ }^{33}$ In an open-label feasibility study by Carhart-Harris et al., ${ }^{34}$ participants with moderate to severe treatment-resistant depression (averaging 17.7 years and 4.6 failed treatments) were treated with two oral doses of synthesized psilocybin seven days apart (10 mg and $25 \mathrm{mg}$ ), with psychological support provided before, during, and after treatments. Participants who completed all treatments $(n=19)$ showed reduced depression scores at all post-treatment time points including the final six-month follow-up. No serious adverse events were reported and treatment was well tolerated. Common side effects were transient anxiety (79\%), headaches ( $42 \%$, duration $<2$ days), and transient nausea without vomiting (26\%). Notably, reductions in depressive symptoms were predicted by the quality of the acute psychedelic experience; the best clinical outcomes (measured at 5 weeks) were related to strong "mystical-type" experiences during the psilocybinassisted sessions. ${ }^{34}$ In a qualitative thematic analysis of interviews at the six-month follow-up, participants described their experiences as an "expansion" or "rebooting" of the mind, which enabled "a change from disconnection (from the self, others, and the outside world) to connection and ... from avoidance of difficult emotions and memories to acceptance [of them]. ${ }^{, 35}$ This clinical trial was limited by a lack of placebo or control group, but the reported safety and efficacy outcomes suggest that further investigation into psilocybin-assisted therapy for the treatment of depression is warranted. Following the designation of Breakthrough Therapy, clinical trials are currently underway by COMPASS Pathways $^{32}$ and the Usona Institute. ${ }^{33}$

\section{Ayahuasca, N,N-dimethlytryptamine, and Depression}

Similar to the preliminary findings of psilocybin, ayahuasca has also been found to have potential as a treatment for depression. ${ }^{14}$ Ayahuasca is a South

J Mental Health Addic Nurs Vol 4(1):e1-e13; February 19, 2020.

This article is distributed under the terms of the Creative Commons Attribution-Non

Commercial 4.0 International License. (C) Denis-Lalonde and Estefan. 
American sacramental plant-based beverage containing several psychoactive compounds, most notably N,Ndimethyltryptamine (DMT). DMT occurs naturally in many plants and is endogenous in animals (including humans), though its source and effect within the human body are not fully understood. ${ }^{36}$ In several studies in both clinical and ceremonial contexts, participants consistently demonstrated significantly decreased depressive symptoms following treatment with ayahuasca as measured with a variety of validated depression scales. ${ }^{14}$ In an open-label trial of six participants with mild, moderate, or severe depression, a one-time dose of ayahuasca produced statistically significant reductions in depressive scores $(p<.01) .{ }^{37}$ The only reported adverse event, vomiting, affected half the participants, though they did not feel that this caused severe discomfort during the experience. Clinical research of intravenous administration of DMT has demonstrated a high safety profile, low incidence of adverse events, and a short duration of effects (5-15 minutes); results show participants had powerful mystical experiences which may have therapeutic potential for treatment of depression, PTSD, alcoholism, obsessive-compulsive disorder, and in psychological/spiritual work with the dying. ${ }^{22}$

\section{Ketamine, Depression, and Anxiety}

Ketamine has been used off-label as a treatment for depression (with or without psychotherapy) for several decades. Ketamine is a legally available dissociative anesthetic with psychedelic properties, and it is included in the World Health Organization' ${ }^{38}$ list of essential medicines. Ketamine is a safe and effective anesthetic and a fast-acting antidepressant; it has also been used in conjunction with psychotherapy for the treatment of PTSD, obsessive-compulsive disorder, bipolar I and II disorder, and other psychiatric conditions. ${ }^{26}$

Though protocols vary by practitioner and patient, treatment may include several sessions in short succession for acute symptoms or maintenance treatments as needed. In a retrospective analysis of data from three U.S. psychiatric practices using ketamine-assisted psychotherapy, Dore et al. ${ }^{26}$ demonstrated clinically significant improvements from baseline in depression and anxiety scores $(p<.0001)$ in persons with $3-5$ years of psychotherapy treatment and taking an average of 2.84 psychiatric medications for moderate depression and anxiety $(n=235)$. Those with a higher number of ketamine-assisted visits as well as those with the most severe symptom burden (including suicidality) showed the greatest improvements. ${ }^{26}$ No patients experienced a physical dependency for ketamine, nor was any drug-seeking behavior observed following treatment. In Dore et al.'s ${ }^{26}$ study, fewer than 15\% of participants experienced adverse effects such as nausea and vomiting.

A patented intranasal version of ketamine, Esketamine, was approved by the FDA in March 2019 for treatment-resistant depression. ${ }^{2}$ Although it remains to be determined which of these medications and treatment protocols will be most effective for treating depression, Doblin ${ }^{2}$ stressed that "while ketamine administration may be sufficient to relieve some kinds of symptoms, the combination of the drug with psychotherapy might produce lasting changes." 2 This aligns with the wider and developing perspective that psychotherapy (or psychological support) is a valuable, even integral component of psychedelic-assisted therapies.

\section{Microdosing and Well-Being}

Taken semi-regularly in minute, barely perceptible doses known as 'microdoses,' some psychedelics are being used in non-clinical contexts by individuals who report experiencing enhanced mental health, creativity, and well-being. In the first worldwide open enrollment study, participants followed a 1-month microdosing protocol with self-acquired LSD or psilocybin and self-reported their results using a validated mood assessment tool. ${ }^{39}$ Preliminary results showed $80 \%$ of participants $(n>1000)$ reported positive or neutral experiences with microdosing; common experiences included elevation in mood, increased productivity and creativity, and better social relationships. As there are extensive limitations to open, exploratory research designs, several trials are currently underway to assess the physical and mental changes that occur when participants are given small measured doses of pharmaceutical-grade LSD and psilocybin, or a placebo, in a clinical setting. ${ }^{39}$

\section{Psilocybin, LSD, and End-of-Life Care}

Anxiety and psychological distress can arise for people with terminal and life-limiting diagnoses. Currently, limitations in spiritual care options for patients 
with advanced serious illness means that existential distress and spiritual needs may go unmet. ${ }^{24}$ Although research in the 1950s and 1960s examined the use of psychedelics in this context, palliative care has seen the re-emergence of psychedelic research as a means to alleviating distress for the dying. ${ }^{40}$ In 2016, two landmark studies using psilocybin to treat cancerrelated depression and anxiety were published. In a randomized double-blind, crossover trial, participants with life-threatening cancers who had received a high dose of psilocybin with psychological support $(\mathrm{n}=46)$ experienced significant $(p<.001)$ and lasting decreases in symptoms of depression and anxiety and an increase in quality of life. ${ }^{41}$ At the 6-month follow-up, these results were sustained: about $80 \%$ of patients continued to show clinically significant decreases in depression and anxiety symptoms. In a second double-blind, controlled trial $(\mathrm{n}=29)$, a single active dose of psilocybin in conjunction with psychotherapy produced immediate and sustained anxiolytic and antidepressant effects in patients with cancer-related psychological anguish, including a decrease in existential distress and an increase in reported spiritual well-being. ${ }^{42}$ No serious adverse events were reported in these two trials, and transient adverse events such as anxiety, nausea, and hypertension resolved by the end of the sessions.

In a randomized, double-blind, active placebocontrolled pilot study $(\mathrm{n}=12)$, Gasser et al. ${ }^{43}$ demonstrated that LSD can reduce anxiety associated with life-threatening illness when administered in a safe and supervised setting with psychological support. Though these findings are preliminary, they support the need for ongoing psychedelic research in palliative care. Although these psychedelics are currently illegal substances, there is increasing evidence of therapeutic benefit. As Carhart-Harris and Goodwin ${ }^{8}$ noted, "it seems [. . .] paradoxical, even incredible, that such drugs should not be available for medical use in conditions for which euthanasia is already available." ${ }^{8}$ The absence of effective treatments for spiritual distress and the expanding access of medical assistance in dying (MAID) may indicate a turning point for palliative care practitioners to consider psychedelic-assisted therapies as a means to address mental health and spiritual care needs.

\section{Psychedelics and Alcohol Dependence}

The use of a drug-facilitated experience to treat problematic use of another drug seems counterintuitive. ${ }^{44}$ Research into the use of psychedelics to address substance use, however, has a long history. In the 1950s, promising research into LSD to treat problematic alcohol use was conducted. ${ }^{6}$ A meta-analysis of six randomized controlled trials conducted in the $60 \mathrm{~s}$ and 70 s concluded that participants with chronic alcohol dependence who were treated with a single high dose of LSD were almost twice as likely to have reduced their alcohol use for up to 12 months post-treatment as compared to the control group ( $p<.0005)$, including maintained abstinence from alcohol at 3 months post-treatment in some trials. ${ }^{45}$ In these combined trials $(n=536), 8$ adverse events were reported, none with lasting harmful effects.

In a small proof-of-concept study examining the safety and preliminary efficacy of psilocybin use for alcohol dependence, Bogenschutz et al. ${ }^{46}$ noted significant decreases in drinking days following psilocybin-assisted therapy, which was largely sustained at the final 9-month follow-up $(n=10)$. Notably, a correlation was found between stronger mystical-type experiences and decreased alcohol use, and no lasting adverse events were reported. As it is not common for psychiatric drugs to have a lasting effect following a single dose, Krebs and Johansen ${ }^{45}$ recommended further exploration into this treatment for alcohol dependence, including repeated doses and the exploration of other psychedelics. It has also been discussed that MDMAassisted therapy may be helpful for the treatment of trauma-induced alcohol dependence and substance use disorders. ${ }^{47}$ An open-label proof-of-concept study demonstrated the safety and tolerability of an 8-week course of MDMA-assisted psychotherapy for alcohol use disorder, with longer-term outcomes in drinking behavior to be published upon completion of the study. ${ }^{18}$ Additionally, observational and anthropological studies examining religious and spiritual communities who regularly partake in ceremonial use of ayahuasca or peyote (a cactus containing mescaline) demonstrated consistently lower rates of substance use, including alcohol, in these groups. ${ }^{1,9}$ 


\section{Psilocybin and Tobacco Cessation}

In the first-ever study exploring the use of psychedelics as an adjunct to tobacco cessation treatment, 15 participants with an average of 6 previous attempts at quitting and 31 years as smokers underwent 2-3 psilocybin-assisted sessions as part of a 15 -week treatment protocol. ${ }^{48}$ In this open-label pilot study, the psychological and biological assessment showed 80\% of participants were smoking abstinent at the 6-month follow-up. In a long-term follow-up study of these participants, $67 \%$ were still abstinent at the 12 -month follow-up and $60 \%$ at the $\geq 16$-month follow-up. A majority (86.7\%) of participants felt their psilocybin experience was "among the five most personally meaningful and spiritually significant experiences of their lives. ${ }^{.49}$ In a qualitative follow-up study which interviewed these same participants an average of 30 months after their treatment, the psilocybin-facilitated experiences of interconnectedness, awe, and curiosity, along with preparatory counselling, rapport-building, and reduced tobacco withdrawal symptoms were reported as central to successful smoking cessation. ${ }^{13}$ With no reported clinically significant adverse events (transient experiences of fear, headaches, and elevated systolic blood pressure resolved within hours of the psilocybin sessions), these preliminary findings suggest greater success than conventional behavioral and pharmacological treatments for tobacco dependence, which typically have $<35 \%$ success rate. ${ }^{48}$

\section{Psychedelics and Opioid Dependence}

Ibogaine is an alkaloid derived from the root of an African shrub, Iboga. Ibogaine has been used to treat opioid dependence for decades and evidence of its efficacy is growing. ${ }^{50,51}$ In an observational study, 30 people with an average of 3 previous opioid dependence treatment attempts were treated with ibogaine for daily opioid use. ${ }^{50}$ Half of the participants reported no drug use at the 1-month follow-up, with no clinically significant adverse effects. Several pharmacological theories exist to explain the drug's efficacy in attenuating opioid cravings and withdrawal symptoms; however, individuals treated with ibogaine also reported a powerful sense of meaning as a result of their intense ibogaine-facilitated treatment which reduced or eliminated their opioid dependence. ${ }^{51}$
In a double-blind, randomized, active-placebo controlled trial of 70 long-term heroin users who were treated with either low dose or high dose ketamineassisted psychotherapy, those treated with high dose ketamine had significantly greater rates of abstinence $(p<.05)$ and reduced cravings at the 2-year follow-up. ${ }^{52}$ Consistent with other ketamine-assisted therapies, there were no reported adverse events (beyond an acute increase in blood pressure during the session) nor was any consequent ketamine dependence reported.

\section{IMPLICATIONS FOR NURSING PRACTICE}

Existing commentary and emerging research into psychedelic-assisted therapies suggests that nurses have had and will continue to have an important role to play in psychedelic-assisted research and treatment. The current psychedelic renaissance promises new treatment approaches that can benefit people who experience mental illness and addictions. This promise extends into the development of new roles and practices for nurses. Nurses are healthcare leaders in practice development, ethics of care, research, patient and caregiver education, and patient and health system advocacy. Nurses are, therefore, well suited to be leaders in the development of psychedelic-assisted therapies.

\section{LEADERSHIP IN PRACTICE}

As the demand for psychedelic-assisted therapy undoubtedly increases, there will be a need for trained, competent, and experienced practitioners, as well as a governing body, to oversee the licensing of individuals involved in this work. ${ }^{9}$ As a self-governing profession emphasizing public good, evidence-based practice, and collaborative models of care, nurses are appropriately primed for this work that demands a high degree of professionalism, responsibility, interpersonal skills, and ethical integrity. Phelps developed six competencies for the training of psychedelic practitioners: "empathic abiding presence, trust enhancement, spiritual intelligence, knowledge of the physical and physiological effects of psychedelics, self-awareness and ethical integrity, and proficiency in complementary techniques." 11 These competencies are strongly aligned with the values and practices of nurses, particularly in contexts that have integrated 
spiritual care and complementary techniques, such as palliative and holistic care. ${ }^{24}$

Due to their proximity to patients and the emphasis on the development of therapeutic, trusting relationships, nurses have historically been the professionals who remain at the bedside, 'hold space', and serve as a supportive witness to the complex narratives of patients' lives. ${ }^{24}$ As such, nurses occupy a unique position of trust, which is critical for the safe and meaningful integration of psychedelic-assisted experiences. These trusting, therapeutic relationships create opportunities for care for patients and health providers. Penn et al. ${ }^{28}$ argued that these relationships enable authenticity, self-compassion, and healing. People undergoing psychedelic-assisted therapy also require physical assessment and monitoring during treatment. In addition to spiritual and other perhaps intangible aspects of nursing care, nurses are also highly educated health practitioners with knowledge and skill in physical and psychological assessment and intervention. Nurses can provide psychological support to persons undergoing psychedelic-assisted therapies; they are also able to screen and monitor patients and intervene in the event of an adverse reaction or medical emergency. With this dual-mandate of medical and spiritual care, nurses are uniquely qualified to become psychedelic practitioners and to take a leadership role in the provision of psychedelicassisted therapies.

\section{LEADERSHIP IN ETHICS AND RESEARCH}

The introduction of psychedelic-assisted therapies calls forth obligations for ethical practice and meaningful research inquiry. If psychedelic-assisted therapy is to become an available treatment option, ongoing ethical oversight will be needed to ensure that practitioners and researchers comply with ethical standards, and are being appropriately trained and governed. ${ }^{1,9}$

The literature shows that psychedelic-assisted therapy research is multi-dimensional. The focus on 'set and setting', psychological support, and integration, suggest that research needs to extend beyond randomized-controlled drug trials and pharmaceutical development. Nurses possess expanded knowledge of and insights into the impacts of social determinants on individual and population health. In the past, psychedelic trials have typically lacked diversity in both researchers and participants, including minority groups such as autistic adults, LGBT persons, and people of colour; this presents an ethical concern as trial outcomes may not be generalizable to these populations. Consequently, some groups may be denied access to treatment or provided inappropriate treatment, further deepening systemic inequities to health and well-being for minority populations. ${ }^{53,54}$ As such, nurses can bridge gaps between the production of evidence for treatment and service provision that alleviates suffering for those who need it. With a professional mandate to promote health, prevent illness, restore health, and alleviate suffering ${ }^{55}$ nurses will need to advocate in practice, research, and leadership contexts to ensure that psychedelic-assisted therapies are ethically and inclusively researched and enacted.

Also, the increasing 'medicalization' of psychedelics creates ethical tensions between effective service provision and for-profit pharmaceutical ventures. These include concerns of exaggerated efficacy claims and conflicts of interests over the production of psychedelic research and knowledge on behalf of the pharmaceutical industry. ${ }^{56}$ It is also possible that the sanctioning of psychedelics for medical purposes may embolden some to use these potent drugs for self-treatment, particularly if there is limited access to psychedelic-assisted therapy in controlled, supportive contexts. As seen today in recreational contexts, a harm reduction education approach would be appropriate in limiting adverse effects from the quasi-therapeutic use of psychedelics. There is also an ethical imperative to ensure that people who use these drugs, whether in recreational contexts or more traditional, ceremonial settings, are not criminalized under this new paradigm. A cautious and diligent yet steadfast approach is required to ensure the ethical integrity of the psychedelic renaissance in both research and practice.

\section{LEADERSHIP IN POLICY, ADVOCACY, AND EDUCATION}

For nurses in influential positions in government, educational institutions, and other policy and practice decision-making roles, there is a need to re-examine the evidence for psychedelic-assisted therapies that

J Mental Health Addic Nurs Vol 4(1):e1-e13; February 19, 2020.

This article is distributed under the terms of the Creative Commons Attribution-Non

Commercial 4.0 International License. (C) Denis-Lalonde and Estefan. 
should be unhindered by social and political pressures which have inhibited past research and treatment development. Key among the challenges nurses face are prohibitionist drug policies worldwide. These policies hinder research by unduly limiting access to psychedelics drugs; they also reinforce the "moral panic" and stigma associated with these drugs, the people who might benefit from using them, and those wanting to study them. So while the primary aim of these emerging psychedelic-assisted therapies is to alleviate human suffering, more broadly it may also be "the thin end of a wedge that moves drug policy away from the elusive goal of eradication in favor of more nuanced strategies that harness the benefits of psychotropic drugs while minimizing their risks." 57

In the current psychedelic renaissance, policy related to regulation, treatment, and harm reduction will need to be guided by public health principles. Haden et al. ${ }^{9}$ proposed a public-health-based model for the regulation of psychedelic drugs drawn from lessons learned from alcohol and tobacco regulation. This model includes considerations of governance, supervision, youth access, supply control, demand mitigation, and evaluation. The lessons learned from the legalization and regulation of cannabis in many jurisdictions will also help inform approaches to psychedelic drug regulation. Nurses are strongly positioned to lead and develop public-health-informed initiatives to guide the implementation, delivery, and evaluation of psychedelic-assisted therapies. Nurses are established policy and advocacy leaders, yet the health system does not make full use of this aspect of registered nurses' scope of practice. ${ }^{58}$ Nurses can advocate for the expansion of innovative and evidencebased treatments that support the health and well-being of stigmatized populations, such as those with mental illness or addictions. For example, Argento, Tupper, and Socias ${ }^{59}$ surmised that psychedelic-assisted therapies might be a missing link to a successful, multifaceted response to the opioid overdose crisis in North America.

Accountable, professional care and advocacy have their roots in professional and pre-licensure education. As psychedelic-assisted therapies become more available, the curricula of professional programs in psychiatry, medicine, social work, and nursing will need to be adapted. The next generation of health and social care providers will need to be open to these new options for mental health care, palliative care, and addiction treatment while navigating ethical, social, and political pressures. It would behoove nurse educators, professors, academics, and clinicians to remain current and responsive to this emerging field. If we do not, nurses will lose opportunities to investigate, develop, and practice in the context of psychedelicassisted therapies. The absence of a nursing voice in this context will represent a loss for patients. Nurses' knowledge and skills are valuable clinical and social resources for the development and enactment of psychedelic-assisted therapies.

\section{CONCLUSION}

The emergence of psychedelic-assisted therapies, the psychedelic renaissance, is an unprecedented opportunity for nurses to support the health and wellbeing of persons with complex mental health challenges and addiction disorders. By framing psychedelic-assisted therapy as an issue that aligns with the values and competencies of the nursing profession, the context is created for nurses to participate in interdisciplinary research and practice to advance these innovative approaches to care. As such, there is an opportunity for nurses to cultivate a distinct area of nursing practice, and to be advocates, leaders, innovators, researchers, practitioners, and educators in this field. We must not, however, allow ourselves to be governed by the moralistic and deep-seated sociopolitical ideas about the use of mind-altering drugs - to do so would be to prevent people who are suffering from receiving potentially life-changing treatments. If nurses allow themselves to be left behind, we do a disservice not only to the profession, but also to the patients, clients, and the communities we care for.

\section{DISCLOSURE}

The authors have no conflicts of interest or grant support to disclose.

\section{REFERENCES}

1. Tupper KW, Wood E, Yensen R, Johnson MW. Psychedelic medicine: a re-emerging therapeutic paradigm. Can Med Assoc J 2015;187(14):1054-9. doi:10.1503/ cmaj. 141124 
2. Doblin RE, Christiansen M, Jerome L, Burge B. The past and future of psychedelic science: an introduction to this issue. J Psychoactive Drugs 2019;51(2):93-7. https://doi.org/10.1080/02791072.2019.1606472

3. Grob CS, Greer GR, Mangini M. Editors' introduction: hallucinogens at the turn of the century. J Psychoactive Drugs 1998;30(4):315-9. doi:10.1080/0279107 2.1998.10399707

4. Dyck E. Psychedelic psychiatry: LSD from clinic to campus. Baltimore, MD, US: Johns Hopkins University Press; 2008.

5. Nichols DE. Serotonin, and the past and future of LSD. MAPS Bulletin Special Edition 2013;23(1):20-23. Retrieved from http://www.maps.org/news-letters/ v23n1/v23n1_p20-23.pdf.

6. Mangini M. Treatment of alcoholism using psychedelic drugs: a review of the program of research. J Psychoactive Drugs 1998;30(4):381-418. doi:10.10 80/02791072.1998.10399714

7. Tanne JH. Obituaries: Humphrey Osmond. BMJ. 2004;328(7441):713-4. Retrieved from https:/www .ncbi.nlm.nih.gov/pmc/articles/PMC381240/

8. Carhart-Harris RL, Goodwin GM. The therapeutic potential of psychedelic drugs: past, present, and future. Neuropsychopharmacology 2017;42:1-9. http:// dx.doi.org/10.1038/npp.2017.84

9. Haden M, Emerson B, Tupper KW. A public-healthbased vision for the management and regulation of psychedelics. J Psychoactive Drugs 2016;48(4):243-52. http://dx.doi.org/10.1080/02791072.2016.1202459

10. Meckel F. Guidelines in applying psychedelic therapies. In: Winkelman M, Sessa B, editors. Advances in psychedelic medicine: state-of-the-art therapeutic applications. Santa Barbara, CA, US: Praeger; 2019.

11. Phelps J. Developing guidelines and competencies for the training of psychedelic therapists. J Humanist Psychol 2017;5(5):450-87. doi:10.1177/0022167817711304

12. Greer G, Tolbert R. Subjective reports of the effects of MDMA in a clinical setting. J Psychoactive Drugs 1986;18(4):319-27. doi:10.1080/02791072.1986.1 0472364

13. Noorani T, Garcia-Romeu A, Swift TC, et al. Psychedelic therapy for smoking cessation: qualitative analysis of participant accounts. J Psychopharmacol 2018;32(7):756-69. doi:10.1177/0269881118780612

14. Johnson MW, Hendricks PS, Barrett FS, Griffiths RR. Classic psychedelics: An integrative review of epidemiology, therapeutics, mystical experience, and brain network function. Pharmacol Ther 2019;197:83-102. doi:10.1016/j.pharmthera.2018.11.010

15. Garcia-Romeu A, Richards WA. Current perspectives on psychedelic therapy: Use of serotonergic hallucinogens in clinical interventions. Int Rev Psychiatry 2018;30(4):291-316. doi:10.1080/09540261.2018. 1486289

16. Johnson MW, Richards WA, Griffiths RR. Human hallucinogen research: Guidelines for safety. J Psychopharmacol 2008;22(6):603-620. doi:10.1177/0269881108093587

17. Ot'alora GM, Grigsby J, Poulter B, et al. 3,4-Methylenedioxymethamphetamine-assisted psychotherapy for treatment of chronic posttraumatic stress disorder: A randomized phase 2 controlled trial. J Psychopharmacol 2018;32(12):1295-1307. doi:10.1177/0269881118806297

18. Sessa B, Sakal C, O’Brien S, Nutt D. First study of safety and tolerability of 3,4-methylenedioxymethamphetamine (MDMA)-assisted psychotherapy in patients with alcohol use disorder: Preliminary data on the first four participants. BMJ Case Rep 2019;12(7):e230109. doi:10.1136/bcr-2019-230109

19. Trope A, Anderson BT, Hooker AR, et al. Psychedelicassisted group therapy: A systematic review. J Psychoactive Drugs 2019;51(2):174-188. doi:10.1080/0 2791072.2019.1593559

20. Byock I. Taking psychedelics seriously. J Palliat Med 2018;21(4):417-421. doi:10.1089/jpm.2017.0684

21. Mithoefer MC, Grob CS, Brewerton TD. Novel psychopharmacological therapies for psychiatric disorders: Psilocybin and MDMA. Lancet Psychiatr 2016;3(5):481-88. doi:10.1016/S2215-0366(15)00576-3

22. Strassman R. The Spirit Molecule: A Doctor's Revolutionary Research into the Biology of Near-Death and Mystical Experiences. Rochester, VT: Park Street Press; 2001.

23. Berg L. Notes from a psychedelic research nurse. Newsletter of the Multidisciplinary Association for Psychedelic Studies 1994;5(1). Retrieved from https:// maps.org/news-letters/v05n1/05134nrs.html

24. Rosa WE, Hope S, Matzo M. Palliative nursing and sacred medicine: A holistic stance on entheogens, healing, and spiritual care. J Holist Nurs 2019;37(1):100-106. doi:10.1177/0898010118770302

25. Mithoefer MC, Wagner MT, Mithoefer AT, et al. The safety and efficacy of 3,4-methylenedioxymethamphetamine -assisted psychotherapy in subjects with chronic,

J Mental Health Addic Nurs Vol 4(1):e1-e13; February 19, 2020.

This article is distributed under the terms of the Creative Commons Attribution-Non Commercial 4.0 International License. (c) Denis-Lalonde and Estefan. 
treatment-resistant posttraumatic stress disorder: The first randomized controlled pilot study. J Psychopharmacol 2010;25(4):439-52. doi:10.1177/0269881110378371

26. Dore J, Turnipseed B, Dwyer S, et al. Ketamine assisted psychotherapy (KAP): Patient demographics, clinical data and outcomes in three large practices administering ketamine with psychotherapy. J Psychoactive Drugs 2019;51(2):189-98. doi:10.1080/0 2791072.2019.1587556

27. California Institute of Integral Studies. About the certificate in psychedelic-assisted therapies and research [Internet]. San Francisco, CA, US:Author; 2019. https://www.ciis.edu/research-centers/centerfor-psychedelic-therapies-and-research/about-the -certificate-in-psychedelic-assisted-therapies-and-research

28. Penn AD, Ot'alora M, Raison CL. MDMA-assisted therapy for posttraumatic stress disorder. In: Summers RF, Jeste DV, editors. Positive psychiatry: A casebook. Washington, DC: American Psychological Association; 2019.

29. Wagner AC, Mithoefer MC, Mithoefer AT, Monson CM. Combining cognitive-behavioral conjoint therapy for PTSD with 3,4-methylenedioxymethamphetamine (MDMA): A case example. J Psychoactive Drugs 2019;51(2):166-73. doi:10.1080/02791072.2019.1 589028

30. Multidisciplinary Association for Psychedelic Studies. Press release: FDA grants breakthrough therapy designation for MDMA-assisted psychotherapy for PTSD, agrees on special protocol assessment for phase 3 trials. Santa Cruz, CA, US: Author; 2017. https://maps.org/news/ media/6786-press-release-fda-grants-breakthrough-therapydesignation-for-mdma-assisted-psychotherapy-for-ptsd,agrees-on-special-protocol-assessment-for-phase-3-trials

31. Dos Santos RG, Osório FL, Crippa JA, et al. Antidepressive, anxiolytic, and antiaddictive effects of ayahuasca, psilocybin and lysergic acid diethylamide (LSD): A systematic review of clinical trials published in the last 25 years. Ther Adv Psychopharmacol 2016;6(3):193-213. doi:10.1177/2045125316638008

32. COMPASS Pathways. COMPASS Pathways receives FDA breakthrough therapy designation for psilocybin therapy for treatment-resistant depression: 2018. https:// compasspathways.com/compass-pathways-receivesfda-breakthrough-therapy-designation-for-psilocybintherapy-for-treatment-resistant-depression/

33. Usona Institute. FDA Grants Breakthrough Therapy Designation to Usona Institute's Psilocybin Program for Major Depressive Disorder: 2019. https://www .businesswire.com/news/home/20191122005452/ en/FDA-grants-Breakthrough-Therapy-DesignationUsona-Institutes

34. Carhart-Harris RL, Bolstridge M, Day CM, et al. Psilocybin with psychological support for treatment-resistant depression: Six-month follow-up. Psychopharmacol 2018;235:399-408. doi:10.1007/s00213-017-4771-x

35. Watts R, Day C, Krzanowski J, et al. Patients ' accounts of increased "connectedness" and "acceptance" after psilocybin for treatment-resistant depression. J Humanist Psycho 2017;57(5):520-64. doi:10.1177/0022167817709585

36. Dean JG, Liu T, Hu S, et al. Biosynthesis and extracellular concentrations of n,n-dimethyltryptamine (DMT) in mammalian brain. Sci Rep 2019;9(9333):1-11. doi:10.1038/s41598-019-45812-w

37. Osório F, Sanches R, Macedo L, et al. Antidepressant effects of a single dose of ayahuasca in patients with recurrent depression: A preliminary report. Braz J Psychiatr 2015;37:13-20. doi:10.1590/1516-4446-2014-1496

38. World Health Organization. Model list of essential medicines, 21st list (Licence: CC BY-NC-SA 3.0 IGO). Geneva, Switzerland: Author; 2019. https://www.who .int/medicines/publications/essentialmedicines/en/

39. Fadiman J, Korb S. Might microdosing psychedelics be safe and beneficial ? An initial exploration. J Psychoact Drugs 2019;51(2):118-122. doi:10.1080/027 91072.2019.1593561

40. Dyck E. Psychedelics and dying care: A historical look at the relationship between psychedelics and palliative care. J Psychoact Drugs 2019;51(2):102-107. doi:10 $.1080 / 02791072.2019 .1581308$

41. Griffiths RR, Johnson MW, Carducci MA, et al. Psilocybin produces substantial and sustained decreases in depression and anxiety in patients with life-threatening cancer: A randomized double-blind trial. J Psychopharmacol 2016;30(12):1181-19. doi: $10.1177 / 0269881116675513$

42. Ross S, Bossis A, Guss J, et al. Rapid and sustained symptom reduction following psilocybin treatment for anxiety and depression in patients with life-threatening cancer: A randomized controlled trial. J Psychopharmacol 2016;30(12):1165-80. doi:10.1177/0269881116675512

43. Gasser P, Holstein D, Michel Y, et al. Safety and efficacy of lysergic acid diethylamide-assisted psychotherapy for anxiety associated with life-threatening diseases. J Nerv Ment Dis 2014;202(7):513-520. doi:10.1097/ NMD.0000000000000113

J Mental Health Addic Nurs Vol 4(1):e1-e13; February 19, 2020.

This article is distributed under the terms of the Creative Commons Attribution-Non Commercial 4.0 International License. (c) Denis-Lalonde and Estefan. 
44. Bogenschutz MP. It's time to take psilocybin seriously as a possible treatment for substance use disorders. Am J Drug Alcohol Abuse 2017;43(1):4-6. doi:10.1 080/00952990.2016.1200060

45. Krebs TS, Johansen, P-Ø. Lysergic acid diethylamide (LSD) for alcoholism: Meta-analysis of randomized controlled trials. J Psychopharmacol 2012;26(7):9941002. doi: $10.1177 / 0269881112439253$

46. Bogenschutz MP, Forcehimes AA, Pommy JA, et al. Psilocybin-assisted treatment for alcohol dependence: A proof-of-concept study. J Psychopharmacol 2015;29(3):298-99. doi:10.1177/0269881114565144

47. Sessa B, Higbed L, Nutt D. A review of 3,4-methylenedioxymethamphetamine (MDMA)-assisted psychotherapy. Front Psychiatr 2019;10(138):1-7. doi:10.3389/fpsyt.2019.00138

48. Johnson MW, Garcia-Romeu A, Cosimano MP, Griffiths RR. Pilot study of the $5-\mathrm{HT}_{2 \mathrm{~A}} \mathrm{R}$ agonist psilocybin in the treatment of tobacco addiction. J Psychopharmacol 2014;28(11):983-92. doi:10.1177/0269881114548296

49. Johnson MW, Garcia-Romeu A, Griffiths RR. Long-term follow-up of psilocybin-facilitated smoking cessation. Am J Drug Alcohol Abuse 2017;43(1):55-60. doi:10 $.3109 / 00952990.2016 .1170135$

50. Brown TK, Alper K. Treatment of opioid use disorder with ibogaine: Detoxification and drug use outcomes. Am J Drug Alcohol Abuse 2018;44(1):24-36. doi:10 $.1080 / 00952990.2017 .1320802$

51. Brown TK, Noller GE, Denenberg JO. Ibogaine and subjective experience: Transformative states and psychopharmacotherapy in the treatment of opioid use disorder. J Psychoactive Drugs 2019;51(2):155-65. doi:10.1080/02791072.2019.1598603

52. Krupitsky E, Burakov A, Romanova T, et al. Ketamine psychotherapy for heroin addiction: Immediate effects and two-year follow-up. J Subst Abuse Treat 2002;23:273-83.

53. Danforth AL. Embracing neurodiversity in psychedelic science: A mixed-methods inquiry into the MDMA experiences of autistic adults. J Psychoactive Drugs 2019;51(2):146-54. doi:10.1080/02791072.2019.1 587116

54. Michaels TI, Purdon J, Collins A, Williams MT. Inclusion of people of color in psychedelic-assisted psychotherapy: A review of the literature. BMC Psychiatr 2018;18(245):1-14. doi:10.1186/s12888-0181824-6

55. International Council of Nurses. The ICN code of ethics for nurses. Geneva, Switzerland: Author; 2012. https:// www.icn.ch/sites/default/files/inline-files/2012_ICN_Codeofethicsfornurses_\%20eng.pdf\#targetText=The $\% 20$ ICN\%20Code $\% 20$ of $\% 20$ Ethics, the $\% 20$ standards $\% 20$ of $\% 20$ ethical $\% 20$ conduct.\&targetText $=$ The $\% 20$ nurse $\% 20$ advocates $\% 20$ for $\% 20$ equity,other $\% 20$ social $\% 20$ and $\% 20$ economic $\% 20$ services.

56. Noorani T. Making psychedelics into medicines: The politics and paradoxes of medicalization. J Psychedelic Stud 2019; Ahead of print. doi:10.1556/2054.2019.018

57. Lamkin M. Psychedelic medicine is coming. The law isn't ready. Scientific American; 2019. https://blogs .scientificamerican.com/observations/psychedelicmedicine-is-coming-the-law-isnt-ready/

58. Reutter L, Kushner KE. Health equity through action on the social determinants of health: Taking up the challenge in nursing. Nurs Inq 2010;17(3):269-80. doi:10.1111/j.1440-1800.2010.00500.x

59. Argento E, Tupper KW, Socias ME. The tripping point: The potential role of psychedelic-assisted therapy in the response to the opioid crisis. Int J Drug Policy 2019;66:80-81. doi:10.1016/j.drugpo.2018.11.006

J Mental Health Addic Nurs Vol 4(1):e1-e13; February 19, 2020. 